\title{
DESIGUALDADE DE OPORTUNIDADES NA FORMAÇÃO DE HABILIDADES: UMA ANÁLISE COM DADOS LONGITUDINAIS
}

\author{
Igor Vieira Procópio ${ }^{\dagger}$ \\ Ricardo da Silva Freguglia $\ddagger$ \\ FLÁvia CheIN $\$$
}

\begin{abstract}
Resumo
Este artigo analisa a evolução da desigualdade educacional e das oportunidades ao longo dos quatro primeiros anos do ensino fundamental por intermédio do desempenho nas disciplinas de língua portuguesa e matemática daquelas crianças que participaram do GERES no período de 2005 a 2008. Os principais resultados obtidos mostram um aumento na desigualdade educacional em matemática e um comportamento praticamente estável em português. No que tange à desigualdade de oportunidades, no fim do período analisado, os valores são de $8 \%$ para matemática e $11 \%$ para português. Percebe-se um aumento nesses valores ao longo do período, sendo que português apresenta um crescimento superior ao de matemática, $109 \%$ e $80 \%$, respectivamente. A característica que mais influencia na desigualdade de aprendizado é o nível socioeconômico.
\end{abstract}

Palavras-chave: Desigualdade de Oportunidades; Formação de Habilidades.

\begin{abstract}
The aim of this paper is to analyze the evolution of education inequality and the inequality of opportunities over the first four years of elementary school in Brazil from 2005 to 2008. Using proficiency performance information of pupils in mathematics and language (Portuguese) from GERES database. The main results show an increase in educational inequality in mathematics and stable behavior in Portuguese over the years under review. With regard to inequality of opportunity, the percentages

\footnotetext{
* Agradecemos o apoio financeiro da FAPEMIG, CNPq e Observatório da Educação 2010/INEP/Capes

${ }^{\dagger}$ Universidade Federal de Juiz de Fora. Juiz de Fora, Minas Gerais, Brasil. E-mail: igor.procopio@ufjf.edu.br

‡ Professor adjunto Faculdade de Economia e do Programa de pós-Graduação em Economia da Universidade Federal de Juiz de Fora (UFJF), pesquisador da FAPEMIG e bolsista de Produtividade em Pesquisa do CNPq. Juiz de Fora, Minas Gerais, Brasil. Email:ricardo.freguglia@ufjf.edu.br

$\S$ Professora adjunto Faculdade de Economia e do Programa de pós-Graduação em Economia da Universidade Federal de Juiz de Fora (UFJF), pesquisadora da FAPEMIG e bolsista de Produtividade em Pesquisa do CNPq. Juiz de Fora, Minas Gerais, Brasil. E-mail:flavia.chein@ufjf.edu.br
} 
Desigualdade de oportunidades na formação de habilidades: uma análise com dados longitudinais 327

are $8 \%$ for math and $11 \%$ for Portuguese. The Portuguese inequality presents a higher increase in these percentages $(109 \%)$ than the math inequality $(80 \%)$ over the four years. The feature that most influences the inequality in proficiency performance is the socioeconomic status of the children family.

Keywords: Inequality of Opportunity; Skill Formation.

JEL classification: D31, I21, I24.

DOI: http://dx.doi .org/10.1590/1413-8050/ea110460 


\section{Introdução}

A teoria de Igualdade de Oportunidades, sintetizada no livro de Roemer (1998), vem ganhando espaço nas discussões sobre justiça distributiva e na formulação de políticas públicas voltadas para a redução das desigualdades. De forma sucinta, essa teoria divide os determinantes dos resultados individuais em dois grupos. Um grupo considerado injusto, que são fatores fora do controle do indivíduo, como gênero, raça, origem familiar, local de nascimento, e outro grupo de fatores considerados justos, que engloba os fatores que estão sob controle do indivíduo, como as escolhas autônomas e o nível de esforço. De acordo com Roemer (1998), as desigualdades que são geradas pelos fatores injustos deveriam ser eliminadas, enquanto que as desigualdades de cunho justo deveriam ser mantidas, pois servem de incentivos para o esforço individual.

O Brasil apresenta uma das maiores desigualdades de renda do mundo, fenômeno vastamente documentado na literatura. Desde os anos de 1960, quando se iniciou o levantamento sistemático de informações censitárias no país, os índices de desigualdade encontrados se situam entre os mais altos do mundo, com valores do índice de Gini partindo de 0,535 em 1960, chegando ao ápice em 1990, com 0,607 e reduzindo a 0,527 em 2011 ${ }^{1}$. Apesar da queda nesses índices na última década, o Brasil continua figurando entre os países com maior desigualdade de renda ${ }^{2}$. Essa desigualdade é, em grande medida, relacionada à origem familiar das pessoas, o que indica uma desigualdade de oportunidades.

A evidente associação entre rendimentos e origem familiar faz com que o uso do arcabouço teórico da igualdade de oportunidades seja adequado para a análise das desigualdades no Brasil. O ideal dessa teoria é um mundo em que as características predeterminadas das pessoas não tenham influência em seus resultados. É uma teoria que se encontra no meio do caminho entre as teorias liberais, em que toda a responsabilidade do sucesso individual é atribuída ao próprio indivíduo e, portanto, não deve haver políticas de redistribuição de renda, e teorias de cunho redistributivista, que consideram a sociedade a responsável pelas conquistas individuais, advogando em causa da redistribuição.

Além dos argumentos normativos em favor da igualdade de oportunidades, pode-se argumentar que políticas públicas voltadas para equalizar as oportunidades individuais terão impactos em termos de eficiência econômica. No campo microeconômico, o fato de a teoria da igualdade de oportunidades responsabilizar os indivíduos por parte de seus resultados mantém os incentivos para o esforço individual. Quando o resultado individual é determinado em parte por suas circunstâncias prédeterminadas o indivíduo sabe que mesmo se exercer um alto nível de esforço, a probabilidade de ele alcançar o topo da distribuição de renda é baixa, o que pode levar esse indivíduo a não se esforçar o tanto que ele se esforçaria se soubesse que o principal determinante de sua renda seria seu esforço.

Já no campo macroeconômico, diversos autores, como Bourguignon (1981), Barro (2000), Rebelo (1991), Deininger \& Squire (1998), Galor \& Moav (2004), tentam relacionar desigualdade de renda com crescimento econômico, con-

\footnotetext{
${ }^{1}$ Ver, entre outros, Fishlow (1972), Langoni (2005),Bonelli \& Sedlacek (1988), Hoffman (1989), Lam \& Levison (1990), Ramos (1993), Barros \& Mendonça (1995), Deininger \& Squire (1996), Ferreira \& Litchfield (1996, 1999), Ferreira (2000).

${ }^{2}$ Ver Barros et al. (2006) sobre a queda recente da desigualdade de renda brasileira.
} 
tudo, não conseguem definir precisamente a influência da desigualdade no crescimento. Um recente estudo de Marrero \& Rodríguez (2013) traz uma nova luz à discussão. Os autores argumentam que o efeito da desigualdade no crescimento varia em função de sua natureza. Se a desigualdade é em função de diferentes níveis de esforços empreendidos pelos indivíduos, ela exerce um efeito positivo no crescimento. Entretanto, quando é relacionada às características pré-determinadas dos indivíduos, exerce um efeito negativo no crescimento. Portanto, quanto maior a desigualdade de oportunidades em um país, maiores serão os entraves ao crescimento. A parcela da desigualdade resultante de circunstâncias fora do controle do indivíduo leva a uma acumulação de capital humano subótima e consequente baixo crescimento, ao passo que a parcela em função de diferentes escolhas e nível de esforço incentivam o investimento do indivíduo em capital humano (Ferreira et al. 2005).

Todo o arcabouço teórico da igualdade de oportunidades, comumente aplicado à desigualdade de renda, pode ser aplicado à desigualdade educacional. O estudo das desigualdades educacionais pode ser justificado pela importância da educação por si própria, como promotora do desenvolvimento humano e das capacidades individuais (Sen, 2000), mas também em função de sua relação com a desigualdade de renda. No Brasil, a partir do trabalho de Langoni (2005), alguns estudos começaram a apresentar evidências de que o principal determinante da alta desigualdade é a disparidade educacional de sua população (Barros \& Mendonça 1995, Ferreira 2000). Esses estudos apontam evidências de que a desigualdade de salários apresentada no mercado de trabalho é um reflexo da diferença de produtividade dos trabalhadores, e que essa diferença não é gerada no mercado de trabalho. Barros \& Mendonça (1995) desenvolvem um modelo dividindo o ciclo de vida do trabalhador em dois períodos, um período pré-mercado de trabalho, em que as habilidades são formadas, e um segundo período correspondendo ao mercado de trabalho, no qual as habilidades são transformadas em produtividade e remuneradas pelos salários. Os autores concluem que a maior parcela da desigualdade é gerada no primeiro período do ciclo de vida, ou seja, na fase de formação das habilidades. Portanto, os trabalhadores entram no mercado de trabalho em desigualdade de condições.

Do mesmo modo que a desigualdade de renda no Brasil é fortemente relacionada com a origem social dos indivíduos, as desigualdades educacionais também o são. Diversos fatores contribuem para a formação de habilidades. Alguns desses fatores estão sob o controle dos indivíduos, como motivação e esforço, enquanto outros não podem ser considerados de responsabilidade da criança, como local de residência e origem familiar. Evidências apontam que grande parte da desigualdade de desempenho dos alunos se deve às desigualdades de nível socioeconômico dos alunos. Além da questão socioeconômica, os insumos escolares também exercem influência nas desigualdades educacionais (Albernaz et al. 2002). Em um ambiente com elevada desigualdade de oportunidades educacionais, maiores investimentos podem ser realizados em pessoas com melhor background social em detrimento de pessoas com maior talento inato, podendo ocasionar perda de eficiência no sistema educacional.

Recentes estudos vêm aplicando o arcabouço teórico da igualdade de oportunidades para o campo da educação, entre os quais estão Albernaz et al. (2002), Waltenberg \& Vandenberghe (2007), Gamboa \& Waltenberg (2012), Ferreira \& Gignoux (2014), Diaz (2012). Esses estudos encontram evidências de que parte das desigualdades educacionais é determinada por característi- 
cas relacionadas com a origem familiar. No entanto, tem-se apenas um quadro de momento, pois utilizam dados em cross-section. Não é possível analisar o comportamento da desigualdade e nem da influência das circunstâncias ao longo do período escolar.

O objetivo deste artigo é analisar o comportamento da desigualdade educacional ao longo do período escolar, bem como a desigualdade de oportunidades por intermédio do desempenho nas disciplinas de língua portuguesa e matemática daquelas crianças que participaram do GERES (Projeto Geração Escolar), no qual foi acompanhada uma amostra de crianças durante os quatro primeiros anos do ensino fundamental. Essa base permite avaliar o grau de desigualdade com que as crianças iniciam o período escolar obrigatório bem como sua evolução nos primeiros anos. Dessa maneira, é possível verificar o quanto da desigualdade educacional é gerada antes da entrada na escola e qual o papel da escola nesse processo, ampliação ou redução.

O artigo está estruturado em mais cinco seções além desta introdução. A próxima seção apresenta a teoria da igualdade de oportunidades e sua aplicação no campo educacional. A seção 3 traz uma descrição do banco de dados utilizado. A terceira seção apresenta a estratégia empírica, a quarta as estatísticas descritivas e resultados e a quinta seção apresenta as considerações finais.

\section{Igualdade de Oportunidades Educacionais}

O debate sobre a responsabilidade individual nos resultados econômicos vem ganhando destaque nas discussões de políticas públicas voltadas para a redistribuição de renda. A Theory of Justice (Rawls 1971) pode ser considerado o primeiro trabalho sobre justiça distributiva que separa os determinantes dos resultados em fatores de responsabilidade do indivíduo e fatores fora do controle do indivíduo. Seguindo Rawls, destacam-se os trabalhos de Dworkin $(1981 a, b)$, Arneson $(1989,1990)$ e Cohen $(1989)$. Entre os economistas, Sen (1979), em seu trabalho Equality of What?, parte do pressuposto que toda teoria normativa sobre ordenamento social associa justiça à igualdade de algo. O que diferencia essas teorias é o que se deve igualar. Sen defende que o essencial é a formação das capacidades do indivíduo e que a atenção das políticas públicas deve ser na distribuição dessas capacidades. Roemer (1998) apresenta uma definição formal do conceito de igualdade de oportunidades, estabelecendo de maneira abstrata a distinção entre os fatores sob responsabilidade do indivíduo e as circunstâncias fora do seu controle.

Roemer (1998) formulou seu modelo teórico de forma bastante flexível, tanto na definição dos fatores injustos (que na linguagem da teoria são chamados de circunstâncias) quanto na definição de qual resultado deve ser objeto de equalização das oportunidades. Os primeiros artigos empíricos, com destaque para os estudos de Betts \& Roemer (2005), Roemer et al. (2003), Checchi \& Peragine (2010), focaram a análise na desigualdade de renda.

Apesar de a flexibilidade do modelo desenvolvido em Roemer (1998) permitir a análise de outros resultados individuais além dos rendimentos, a aplicação ao campo educacional requer alguns cuidados. Dois pontos merecem atenção, um relacionado à definição de fatores sob responsabilidade do indivíduo e fatores fora do controle individual, e outro ponto referente à estratégia empírica para a mensuração das desigualdades. 
O principal problema na definição de fatores justos e injustos na aplicação da teoria de igualdade de oportunidades ao campo da educação se refere à idade dos indivíduos. Enquanto que na análise da desigualdade de renda os indivíduos são todos adultos, na avaliação das desigualdades educacionais os indivíduos podem ser adolescentes e até mesmo crianças. A atribuição de responsabilidades a crianças e adolescentes é mais delicada e controversa. Em que medida se pode atribuir a esses indivíduos a responsabilidade por suas escolhas, em especial o nível de esforço e dedicação nos estudos? Será que essa responsabilidade deve ser atribuída à criança e ao adolescente ou aos seus pais e professores? Caso não se possa atribuir responsabilidade a estes indivíduos, toda a desigualdade seria injusta e não haveria a necessidade de decompor a desigualdade em fatores justos e injustos.

Essa discussão é levantada nos artigos de Waltenberg \& Vandenberghe (2007), Peragine \& Serlenga (2007), Gamboa \& Waltenberg (2012). Peragine \& Serlenga (2007) preferem não aprofundar no problema e focam a análise na educação superior, em que os indivíduos já são adultos e, portanto passíveis de serem reponsabilizados por suas decisões. Waltenberg \& Vandenberghe (2007) e Gamboa \& Waltenberg (2012) analisam a desigualdade educacional para adolescentes entre 14 e 15 anos e argumentam que, apesar de não serem plenamente capazes de serem responsabilizados por suas decisões, já são pelo menos parcialmente capazes. Os autores ressaltam que em vários países adolescentes de 16 anos já podem votar e dirigir.

No caso do presente estudo essa questão é mais delicada, pois avaliamos a desigualdade para crianças que têm 6 ou 7 anos no início do período analisado e 10 ou 11 ao final do período. Apesar da dificuldade de se atribuir responsabilidade às escolhas que crianças nessa faixa etária realizam, o arcabouço teórico da igualdade de oportunidades ainda pode ser utilizado. Dentre os principais determinantes do sucesso educacional estão fatores que não são passíveis de interferência de políticas públicas, como habilidades inatas e até mesmo sorte. Com isso, na fração da desigualdade chamada de esforço na linguagem da teoria da igualdade de oportunidades, estão fatores genéticos, sorte e outros fatores não disponíveis no banco de dados, como preferência dos pais pela educação, disponibilidades de recursos didáticos em casa, entre outros. Todo esse grupo de fatores pode ser interpretado como sendo a desigualdade residual. Portanto, a fração atribuída à desigualdade de oportunidades neste estudo é inferior à verdadeira desigualdade de oportunidades.

Quanto às limitações em relação à estratégia empírica, a análise da desigualdade educacional pode ser realizada em termos de anos de estudo ou distorção idade-série. No entanto, não são critérios adequados, pois não incorporam a qualidade do ensino. Uma pessoa com 10 anos de estudo em um país ou município com um bom sistema educacional não pode ser diretamente comparada com uma pessoa com os mesmos 10 anos de estudo em outra localidade com um sistema de ensino inferior. Em função disso, as avaliações educacionais estão sendo realizadas através dos resultados de testes padronizados aplicados em larga escala. Os resultados desses testes podem ser diretamente comparados entre pessoas de diferentes localidades e ao longo do tempo.

A análise das desigualdades com o uso de testes padronizados não pode ser realizada com as mesmas técnicas utilizadas para a análise da desigualdade de renda. Os resultados dos testes são divulgados não em suas notas absolutas, mas com o uso da Teoria da Resposta ao Item, que dentre algumas peculiaridades, realiza uma normalização das notas. Essa característica faz com que as 
medidas usuais de desigualdade, como índice de Gini e Theil, sejam inapropriadas para aplicação na avaliação das desigualdades educacionais (Ferreira \& Gignoux 2014). A seção 4 traz esta discussão em maiores detalhes.

A escolha das circunstâncias a serem consideradas na análise envolve sempre certo grau de arbitrariedade e limitação em função das informações disponíveis. No caso de crianças, tal definição é ainda mais complicada, como mencionado anteriormente. Se considerarmos que uma criança de 7 anos não pode ser responsabilizada por suas escolhas, todos os fatores que determinam o aprendizado seriam considerados circunstâncias. Os principais determinantes para o aprendizado das crianças são os fatores genéticos, as características familiares e características escolares. Neste estudo, utilizamos como circunstâncias o nível socioeconômcio da família, a raça e o gênero da criança e o município da escola.

O nível socioeconômico das famílias é apontado por diversos estudos como o principal fator determinante do sucesso educacional. Desde o relatório Coleman (1966) que a importância do nível socioeconômico é destacada. O nível educacional dos pais influencia no aprendizado das crianças, tanto por transferir uma maior preferência pela educação quanto pela maior capacidade de auxiliar as crianças nas tarefas educacionais levadas para casa. A riqueza da família permite o acesso a melhores escolas e a um maior número de bens que contribuem para o aprendizado, como livros, revistas, computador e acesso à internet. Utilizamos uma variável composta que engloba ao mesmo tempo informações sobre o nível de escolaridade da mãe, a ocupação do pai e a presença de certos bens materiais na casa da criança. A escolha dessa variável em detrimento de se utilizar cada informação separadamente se deu em função de limitações do banco de dados. A próxima seção contém uma explicação da forma de construção desta variável.

Outra característica que aparece como um forte determinante das desigualdades, tanto de renda quanto educacional, é a raça do indivíduo. O Brasil, historicamente, apresenta uma forte desigualdade racial que é facilmente notada no mercado de trabalho. Em média, a população branca possui salários superiores ao recebidos pela população negra. Um dos fatores explicativos desta desigualdade é a discriminação que sofrem os negros em relação aos brancos. Outra explicação reside na menor escolaridade da população negra, tornando-os menos produtivos. Portanto, se faz necessário entender em que momento se inicia a desigualdade entre as raças. Soares et al. (2005) trazem diversos textos investigando a questão da desigualdade educacional entre as raças. Existe uma forte correlação entre raça e nível socioconômico, portanto, parte da desigualdade educacional entre raça pode ser explicada pela circunstância anterior. No entanto, estudos mostram que, mesmo após controlar pelo nível socioeconômico das famílias, as crianças negras ainda possuem um rendimento inferior em comparação com crianças brancas (Soares et al. 2005, Soares \& Alves 2003). Obviamente, a raça da criança não é uma característica de escolha por ela, ou seja, é claramente um problema de oportunidades.

O gênero é um fator importante para a análise da desigualdade de renda. Estudar a desigualdade educacional pode contribuir para um maior entendimento de diferenciais de gênero no mercado de trabalho (Pinheiro et al. 2009). Está bem estabelecido na literatura que as meninas possuem melhor rendimento em português, enquanto que os meninos possuem melhor rendimento em matemática. Neste estudo, é possível avaliar como se dá a evolução desse gap, bem como a sua contribuição para a desigualdade educacional. 
Por fim, o município também pode ser um fator de desigualdade educacional, pois representa, em síntese, a qualidade da rede escolar. Riani \& RiosNeto (2008) apontam que uma boa rede escolar municipal pode reduzir a influência do nível socioeconômico familiar. É importante ressaltar, que neste estudo, apenas quatro municípios compõem a amostra e, com isso, a análise desse fator fica prejudicada. Entretanto, mesmo diante de tal limitação, os resultados considerando essa circunstância serão apresentados, pois não existem estudos mostrando a evolução da contribuição dos municípios para a desigualdade educacional. Mas também serão apresentados resultados apenas com as outras três circunstâncias.

\section{Base de Dados}

A base de dados utilizada neste estudo é proveniente do Projeto Geres, que avalia alunos de uma amostra de escolas durante os quatro primeiros anos do Ensino Fundamental. Portanto, é um estudo longitudinal no qual é possível acompanhar a evolução do nível de habilidades das crianças por meio do nível de proficiência, ou seja, é possível medir a evolução do aprendizado da criança. Ao acompanhar uma amostra de alunos durante a primeira fase do Ensino Fundamental, a análise dos resultados do Geres é uma contribuição para as pesquisas de avaliação educacional no Brasil. Os objetivos do Geres são:

- identificar as características escolares que maximizam a aprendizagem dos alunos e que minimizam o impacto da origem social sobre o aprendizado;

- identificar os fatores escolares que diminuem a probabilidade de repetência dos alunos;

- identificar aquelas características da escola que reduzem a probabilidade do absenteísmo.

O Geres acompanhou alunos entre 2005 e 2008, realizando testes todos os anos, possibilitando estimar os níveis de proficiência em matemática e português. Em 2005, foram aplicados dois testes, um no início do ano, para ser utilizado como diagnóstico, e outro ao final do ano. Ao todo, foram cinco aplicações de testes, denominadas ondas. A onda 1 representa as informações de diagnóstico, a onda 2 as informações relativas à segunda aplicação dos testes, e assim por diante. Foram acompanhados alunos que em 2005 estavam matriculados na primeira série do Ensino Fundamental (ou no segundo ano, onde o Ensino Fundamental já era composto por nove anos). Além da aplicação dos testes, o projeto também aplicou questionários aos professores, diretores, pais e alunos com o objetivo de avaliar o efeito de fatores familiares e escolares no aprendizado. As escolas da amostra estão localizadas em cinco grandes cidades brasileiras - Belo Horizonte, Rio de Janeiro, Campo Grande, Salvador e Campinas, e seis universidades participaram do planejamento, coordenação e execução do projeto - UFMG, PUC-Rio, UEMS, UFBa UNICAMP e UFJF.

As avaliações em larga escala mais difundidas no país, SAEB e Prova Brasil, aplicam testes em crianças a partir da $4^{\mathrm{a}}$ série $/ 5^{\circ}$ ano do ensino fundamental. O Geres inicia a aplicação dos testes para crianças na $1^{\mathrm{a}}$ série $/ 2^{\circ}$ ano, ou seja, crianças mais novas. Esta característica exige alguns procedimentos diferenciados. Primeiro na forma de apresentação do teste para as crianças. Os itens 
não foram apresentados de forma aleatória, como acontece no SAEB. Os itens foram apresentados de acordo com seu nível de dificuldade, do mais fácil para o mais difícil, método considerado mais adequado para as séries inferiores (Soares \& Oliveira 2009). Além dessa ordenação, nas primeiras aplicações do teste, parte dos enunciados foi lida para as crianças.

O segundo procedimento diferenciado se refere à escala de proficiências. Optou-se por uma escala própria de proficiência e não pela escala do SAEB, cujo o grupo de referência é a $8^{a}$ série. De acordo com Soares \& Oliveira (2009), utilizar a $8^{\mathrm{a}}$ série como grupo de referência permite boa comparabilidade para as séries avaliadas no SAEB, mas não produz uma medida adequada para o Geres, dado que as crianças da $1^{\text {a }}$ série estão muito distantes dos alunos da $8^{\text {a }}$ série em termos de habilidades cognitivas. Entretanto, os itens aplicados na $4^{\text {a }}$ série do SAEB 2003 foram calibrados na escala Geres, permitindo que as aproficiências dos alunos participantes do Geres possam ser comparadas com as proficiências do SAEB (Soares \& Oliveira 2009).

A proficiência dos alunos foi estimada com a utilização da Teoria da Resposta ao Item (TRI), método amplamente utilizado nos programas de avaliação educacional (SAEB, Prova Brasil, SARESP, PISA). A TRI permite comparabilidade entre os resultados de testes aplicados em diferentes momentos ${ }^{3}$. A equação 1 apresenta a fórmula para estimação das proficiências.

$$
P\left(Y_{i g}=1 \mid a_{i}, b_{i}, c_{i}, \theta_{j}\right)=c_{i}+\frac{\left(1-c_{i}\right)}{1+e^{-D a_{i}\left(\theta_{j}-b_{i}\right)}}, \theta_{j} \sim N\left(\mu_{g}, \sigma_{g}\right),
$$

onde, $P\left(Y_{i g}=1 \mid\right.$.) representa a probabilidade da resposta $Y_{i g}$ do aluno $j$ do grupo $g$ estar correta dada a proficiência $\theta_{j}$, do aluno, $a_{i}$ representa o parâmetro de discriminação, $b_{i}$ o parâmetro de dificuldade e $c_{i}$ o parâmetro de acerto casual (Soares \& Oliveira 2009).

Após a estimação das proficiências pela fórmula apresentada na equação 1, é realizada uma padronização das notas. A padronização é uma transformação linear das notas originais para uma escala arbitrária, em que existe um movimento de rotação e de translação. Como será visto na seção metodológica, essa transformação impede o uso dos índices de desigualdade comumente utilizados na análise da desigualdade de renda, gini e theil, por exemplo, para a análise da desigualdade educacional. A padronização segue a fórmula apresentada na equação 2 .

$$
y_{i}=\widehat{\mu}+\frac{\widehat{\sigma}}{\sigma}\left(x_{i}-\mu\right)
$$

onde, $y_{i}$ é nota do aluno $i$ após a padronização, $\widehat{\mu} \mathrm{e} \widehat{\sigma}$ representam a média e o desvio padrão arbitrários da distribuição padronizada, respectivamente, $\mu$ e $\sigma$ representam a média e o desvio padrão da distribuição original, respectivamente, e $x_{i}$ é a nota pré-padronização obtida pela aplicação da TRI, conforme equação 1 . No caso do Geres, a média e o desvio padrão arbitrário foram definidos em 100 e 25, respectivamente (Oliveira 2008).

Neste trabalho, além dos níveis de proficiência dos alunos em português e matemática, são utilizadas a raça das crianças, o nível socioeconômico da família e o município de residência, variáveis retiradas dos questionários de pais e alunos para caracterizar a origem familiar.

\footnotetext{
${ }^{3}$ Para maiores detalhes da TRI ver, entre outros, (Baker \& Kim 2004)
} 
O nível socioeconômico é uma variável disponibilizada juntamente com o banco de dados do projeto Geres. É uma variável composta de diversas informações coletadas nos questionários aplicados aos pais e aos próprios alunos. São três dimensões compondo o índice: escolaridade da mãe, ocupação do pai e renda. Esta útlima é composta pela presença de bens de conforto no domicílio, como existência de computador, carro, aparelho de dvd, máquina de lavar, etc ${ }^{4}$.

A tabela 1 apresenta a frequência e percentual das variáveis de raça, gênero e município ${ }^{5}$. Tanto no banco de português quanto no banco de matemática, há uma concentração de crianças pardas, seguidas pelas brancas, negras, indígenas e amarelas, nesta ordem. O gênero está bem equilibrado, com um pouco mais de $50 \%$ de meninos nos dois casos. Em relação aos municípios, Belo Horizonte e Campinas possuem em torno de 30\% cada, Rio de Janeiro $22 \%$ e Campo Grande 16\%. O total de observações é de 5.374 crianças por onda em matemática e 5.281 em português.

Tabela 1: Quantidade e percentual de crianças por raça, gênero e município de residência

\begin{tabular}{|c|c|c|c|c|}
\hline \multirow[t]{2}{*}{ Circunstâncias } & \multicolumn{2}{|c|}{ Matemática } & \multicolumn{2}{|c|}{ Português } \\
\hline & Quantidade & $\%$ & Quantidade & $\%$ \\
\hline \multicolumn{5}{|c|}{ Raça } \\
\hline Branco & 1761 & 32,77 & 1731 & 32,78 \\
\hline Pardo & 2791 & 51,94 & 2735 & 51,79 \\
\hline Negro & 822 & 15,3 & 815 & 15,43 \\
\hline \multicolumn{5}{|c|}{ Gênero } \\
\hline Meninos & 2696 & 50,17 & 2662 & 50,41 \\
\hline Meninas & 2678 & 49,83 & 2619 & 49,59 \\
\hline \multicolumn{5}{|c|}{ Município } \\
\hline Belo Horizonte & 1743 & 32,43 & 1694 & 32,08 \\
\hline Campinas & 1822 & 33,9 & 1811 & 34,29 \\
\hline Campo Grande & 943 & 17,55 & 913 & 17,29 \\
\hline Rio de Janeiro & 866 & 16,11 & 863 & 16,34 \\
\hline Total & 5374 & 100 & 5281 & 100 \\
\hline
\end{tabular}

A tabela 2 mostra a média do nível socioeconômico dos alunos e da proficiência em cada onda de aplicação dos testes. Tanto considerando os alunos que fizeram todos os testes de português como aqueles que fizeram todos os testes de matemática, o nível socioeconômico dos alunos apresenta a mesma média, com uma pequena diferença no desvio padrão. Nota-se uma evolução das proficiências ao longo das ondas, que representa o aprendizado das crianças após o início do ensino fundamental.

\footnotetext{
${ }^{4}$ Para mais detalhes da construção desta variável, ver Soares \& Oliveira (2009).

${ }^{5}$ Esses valores são os mesmos para todas as ondas, pois são características invariantes no tempo
} 
Tabela 2: Média do NSE e Proficiência

\begin{tabular}{lll}
\hline & Matemática & Português \\
\hline NSE & $-0,13$ & $-0,13$ \\
\hline \multicolumn{3}{c}{ Proficiência } \\
\hline Onda1 & 101,37 & 100,60 \\
Onda2 & 129,84 & 121,15 \\
Onda3 & 147,64 & 136,99 \\
Onda4 & 189,63 & 152,47 \\
Onda5 & 233,23 & 164,87 \\
\hline
\end{tabular}

Fonte: GERES.

\section{Estratégia Empírica}

Desde Roemer (1998), diversas metodologias vêm sendo aplicadas para mensurar a desigualdade de oportunidades. Os primeiros trabalhos empíricos buscavam avaliar a desigualdade de oportunidades em relação aos salários e rendimentos do indivíduo. Os trabalhos de Pignataro (2012) e Ramos (2012) trazem uma revisão dos principais métodos utilizados. As principais técnicas utilizadas se baseiam em decomposição de índices de desigualdades, principalmente do Índice de Theil.

No campo das desigualdades educacionais, as mesmas técnicas utilizadas para a desiguldade de renda foram aplicadas (Checchi \& Peragine 2005, Gamboa \& Waltenberg 2012). No entanto, Ferreira \& Gignoux (2014) alertam para o problema de usar tais técnicas na análise da proficiência dos alunos quando estas são disponibilizadas em valores padronizados.

Ferreira \& Gignoux (2014) apresentam as propriedades desejáveis que os índices de desigualdade devem atender.

- simetria: requer que o índice seja insensível a permutações no vetor de interesse;

- continuidade nos valores observados;

- princípio da transferência: requer que o índice seja sensível a uma transferência de alguém no topo da distribuição para alguém na parte inferior da distribuição e vice-versa;

Além dessas três propriedades, mais uma, entre duas outras, é desejável, porém nenhum índice de desigualdade atende as duas simultaneamente.

- invariância de escala: requer que o índice seja insensível à mudança de escala, $I(y)=I(\lambda y), \lambda>0$ e $y$ o vetor de interesse, ou;

- invariância em relação à translação: requer que o índice seja insensível à uma translação do vetor de interesse, $I(y)=I(y+a), a \neq 0$.

Ferreira \& Gignoux (2014) destacam que nenhum índice de desigualdade produz uma medida cardinalmente igual para uma distribuição pré e pós padronização dos resultados de proficiência. Os autores ainda chamam a atenção que esse problema ocorre em função da padronização utilizada nos resultados dos testes, e não em função da aplicação da Teoria da Resposta ao Item. Portanto, esse problema ocorre no GERES da mesma maneira que ocorre em 
outros casos de aplicações de teste, SAEB, Prova Brasil, PISA, entre outros, mesmo o GERES utilizando um número maior de parâmetros na estimação da proficiência.

Entretanto, não produzir uma medida cardinalmente invariante à padronização não é um problema muito grave. O que os autores chamam a atenção é que nenhum índice de desigualdade conhecido é ordinalmente equivalente quando aplicado a distribuições pré e pós padronizadas. Ou seja, ao se comparar a desigualdade entre duas ou mais distribuições, nada garante que o ranking entre essas distribuições se manterá quando o índice for aplicado à distribuição pré padronização e à distribuição pós padronização.

Apesar de não fazermos comparações entre países, como Gamboa \& Waltenberg (2012) ou regiões, neste trabalho fazemos comparações entre distribuições ao longo do tempo. Aplicando os conhecidos índices de desigualdade nessas distribuições, já padronizadas, não poderemos ter confiança se a evolução observada nos índices reflete a verdadeira evolução na desigualdade de proficiência. Como solução, os autores sugerem a aplicação de uma medida simples, a variância. Gamboa \& Waltenberg (2012) mostram que a variância aplicada à distribuição pós padronizada é apenas uma tranformação monotônica da variância aplicada à distribuição pré padronização, o que permite uma equivalência ordinal entre as duas distribuições. Portanto, neste trabalho utilizamos a variância aplicada à distribuição da proficiência em matemática e português para as cinco ondas, permitindo acompanhar a evolução da desigualdade de proficiência nos primeiros anos do Ensino Fundamental para uma amostra de crianças.

Após calcular a evolução da desigualdade de proficiência, o nosso interesse recai sobre o quanto dessa desigualdade pode ser explicada por características do indivíduo fora de seu controle. As técnicas utilizadas se baseiam em decomposições da desigualdade. A desigualdade de oportunidades é medida como a relação entre a desigualdade entre os grupos definidos e a desigualdade geral. Seguimos o método usado por Ferreira \& Gignoux (2014), que sugerem uma alternativa paramétrica baseada numa regressão estimada por Mínimos Quadrados Ordinário (MQO), com a proficiência sendo explicada pelas características não controladas pelo indivíduo. O índice de desigualdade de oportunidades será a razão entre a variância explicada pela regressão e pela variância total da proficiência, que é simplesmente o $\mathrm{R}$ quadrado da regressão. É importante destacar que, nesse tipo de construção da desigualdade de oportunidades, a regressão utilizada não pretende estimar relações causais, e o valor do $\mathrm{R}$ quadrado, irá fornecer uma medida subestimada da desigualdade de oportunidades.

Para um melhor entendimento do comportamento da desigualdade de oportunidades, os resultados são apresentados com a inclusão gradativa das circunstâncias, até o resultado final onde as quatro circunstâncias escolhidas são consideradas conjuntamente. A medida é aplicada para as cinco ondas do GERES permitindo uma avaliação da evolução da desigualdade de oportunidades durante os primeiros anos do período escolar.

Ferreira \& Gignoux (2014) destacam algumas vantagens de se utilizar o R quadrado como medida de desigualdade de oportunidades. Entre elas está a possibilidade de decomposição da medida nos componentes individuais utilizados na regressão. Com isso é possível calcular a contribuição marginal de cada circunstância na desigualdade de oportunidades e consequentemente a evolução ao longo do tempo destas características. Os autores sugerem que o 
R2 seja decomposto com o uso da decomposição de Shapley-Shorrocks.

\section{Estatísticas Descritivas e Resultados}

Esta seção apresenta primeiramente gráficos mostrando a evolução da proficiência para os grupos dentro de cada circunstância, ou seja, como se dá a evolução da proficiência comparativamente entre homens e mulheres, entre as diferentes raças dos alunos, entre os diferentes municípios e entre os quintis de nível socioeconômico. Em seguida, são apresentados os resultados para a evolução da desigualdade de proficiência, utilizando a variância como medida de desigualdade, e por fim os resultados dos exercícios econométricos, em que é possível visualizar a evolução da desigualdade de oportunidades e da contribuição de cada circunstância.

Antes de mostrarmos a evolução da proficiência para cada circusntância, destacamos a evolução da proficiência média de cada disciplina, mostrada no gráfico 1. Como se pode perceber, as duas proficiências começam com valores próximos, mas vão se distanciando ao longo das ondas. A proficiência em matemática cresce a taxas crescentes, enquanto que a proficiência em português cresce a taxas decrescentes. Esse fato também foi destacado em Brooke et al. (2014), que utilizaram os mesmos dados do que os utilizados neste artigo. Os autores ressaltam que a diferença entre os padrões das duas disciplinas é algo conhecido na literatura da educação. O aprendizado de português se dá antes mesmo da entrada da criança na escola, devido ao uso da língua na convivência social. Já o aprendizado de matemática é realizado fundamentalmente dentro do ambiente escolar, portanto, sua evolução é mais pronunciada após o início de período escolar. É possível notar que o crescimento do aprendizado em matemática se torna mais expressivo entre as $3^{\mathrm{a}}$ e $4^{\mathrm{a}}$ ondas, que representam as $2^{\mathrm{a}}$ e $3^{\mathrm{a}}$ séries. Esse aumento da inclinação a partir da $3^{\mathrm{a}}$ série é compatível com o processo de ensino das duas disciplinas. Os dois primeiros anos do ensino fundamental (ou os 3 primeiros, quando considerado o ensino fundamental de 9 anos) é voltado mais para o processo de alfabetização, sendo que a partir da $3^{a}$ série aumenta a ênfase no ensino em matemática. Essa diferença entre o comportamento das duas disciplinas é importante para a análise da evolução das desigualdades de oportunidades e da contribuição de cada circunstância.

A figura 2 apresenta a evolução das proficiências em matemática e português, respectivamente, para cada raça conforme declarada nos questionários do Geres. Tanto para a proficiência em português quanto em matemática, as curvas para brancos e pardos evoluem de forma semelhante, mantendo-se a distância entre elas praticamente constante ao longo dos anos. Já a curva de proficiência para negros, além de já começar o período com uma distância maior em relação às outras duas curvas, aumenta esta distância ao longo do período analisado, indicando que a escola não está sendo capaz de reduzir a desigualdade entre as raças, e de forma mais preocupante, está contribuindo para um aumento destas desigualdades. O resultado reforça outras evidências já documentadas na literatura, como Soares et al. (2005); Soares \& Alves (2003).

A figura 3 apresenta a evolução dos níveis de proficiência para cada quintil de nível socioeconômico. Da mesma forma como acontece com a raça, o formato das curvas é semelhante para cada quintil e a distância entre as curvas 


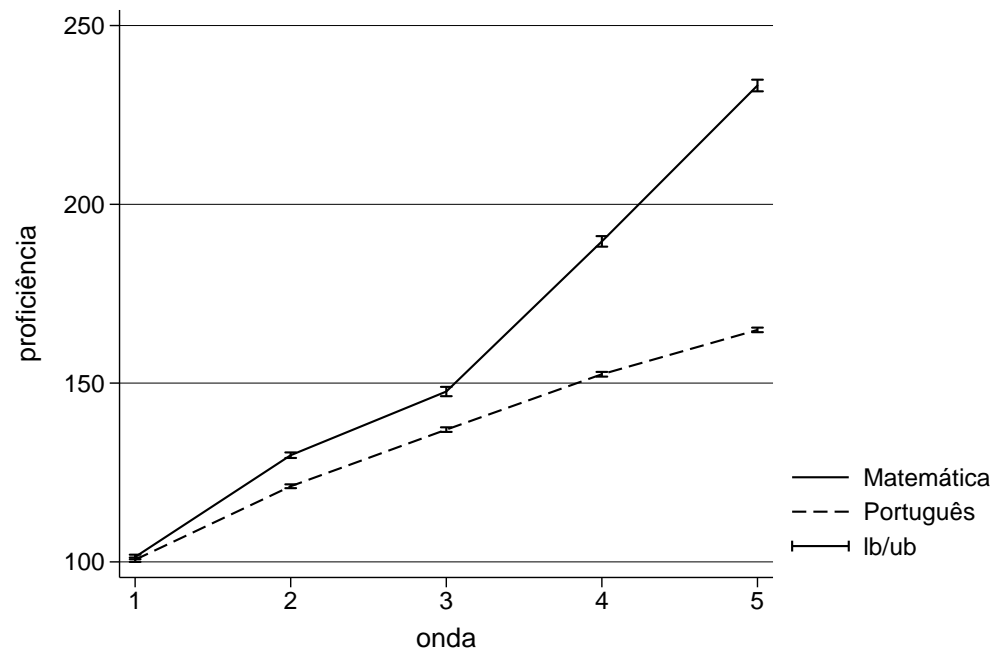

Figura 1: Evolução da proficiência por disciplina

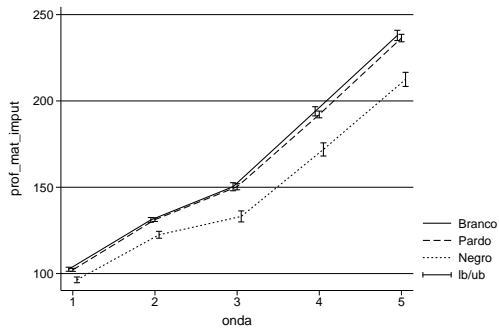

Matemática

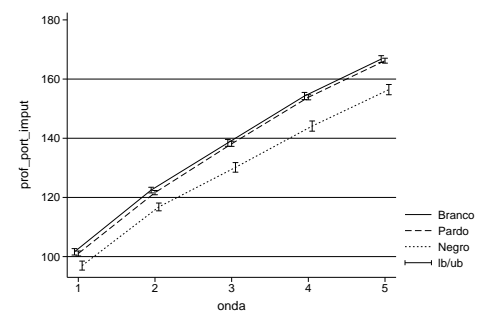

Português

Figura 2: Evolução da proficiência por raça

na proficiência em matemática vai se ampliando, ou seja, a desigualdade entre os grupos socioeconômicos se amplia ao longo dos primeiros anos do Ensino Fundamental.

A figura 4 mostra a evolução da proficiência média por município em matemática e português, respectivamente. Nota-se uma evolução dos níveis de proficiência para todos os municípios e que as linhas não se cruzam, ou seja, os municípios mantêm suas posições relativas iniciais. Mas a distância entre as linhas se altera. Na proficiência em português, a distância entre os municípios se reduz enquanto em matemática a distância se altera menos. Destaque para os municípios de Belo Horizonte e Campo Grande, que iniciam praticamente com o mesmo nível de proficiência, no entanto, na última onda, esta distância é visualmente perceptível, ou seja, as escolas de Belo Horizonte apresentam um maior nível de aprendizado do que as escolas de Campo Grande, este efeito é mais acentuado na proficiência em matemática. Este mesmo efeito ocorre entre os municípios do Rio de Janeiro e de Campinas. Campinas inicia o período com média de proficiência inferior à média apresentada pelo 


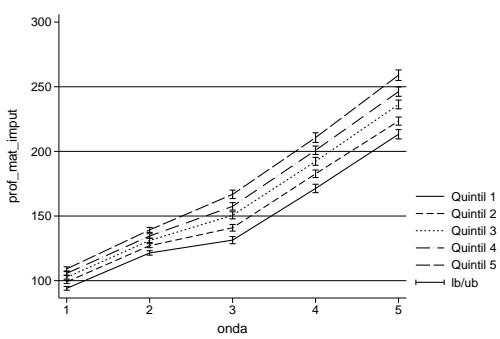

Matemática

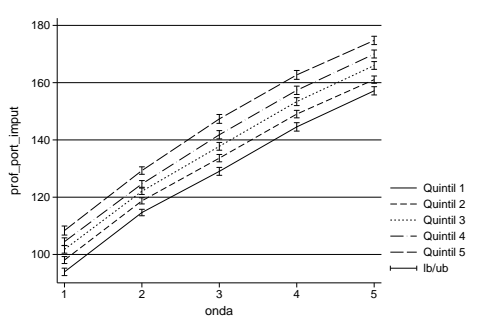

Português

Figura 3: Evolução da proficiência por quintil de nível socioeconômico

município do Rio de Janeiro, mas ao final do período apresenta resultados estatisticamente iguais.

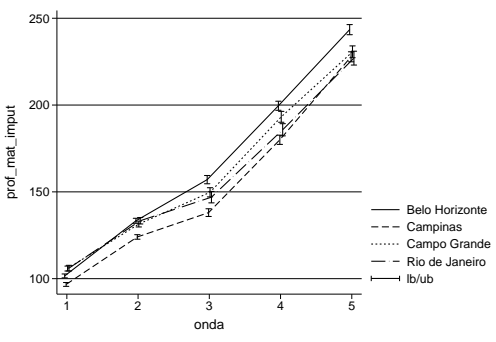

Matemática

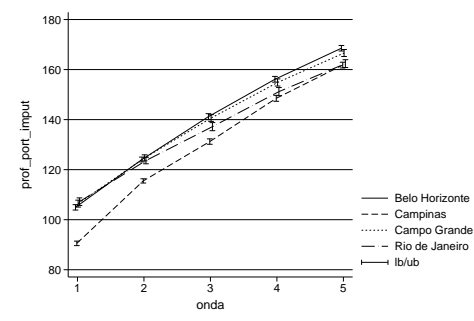

Português

Figura 4: Evolução da proficiência por municípios

A figura 5 apresenta a evolução da proficiência para meninos e meninas separadamente. Para matemática, é possível perceber que no início do período escolar não havia diferenças significativas entre os dois grupos, permanecendo assim nas ondas dois e três. Já na quarta onda, as meninas apresentam uma proficiência média ligeiramente superior aos meninos, consolidando esta tendência na quinta onda. Vale ressaltar que esse resultado não retrata a realidade do Brasil, onde, de acordo com resultados da Prova Brasil, os meninos têm notas em matemática ligeiramente superior na quarta série. Para a proficiência em português, o resultado é semelhante ao observado no Brasil, ou seja, as meninas apresentam uma proficiência superior à dos meninos. No caso do GERES, essa diferença aumenta ao longo dos anos. Esse resultado é importante, pois, sugere que a desigualdade de renda observada no mercado de trabalho entre homens e mulheres não decorre das desigualdades educacionais.

A tabela 3 mostra o comportamento da desigualdade geral de proficiência nos quatro primeiros anos do Ensino Fundamental. O resultado para a onda um mostra que as crianças já iniciam o período escolar com uma desigualdade de conhecimentos, com valores próximos em ambas as proficiências. No entanto, a evolução da desigualdade é muito diferente para matemática e português. Enquanto que em português existe uma quase estabilidade da medida de desigualdade, com uma elevação de apenas $5 \%$, para matemática, 


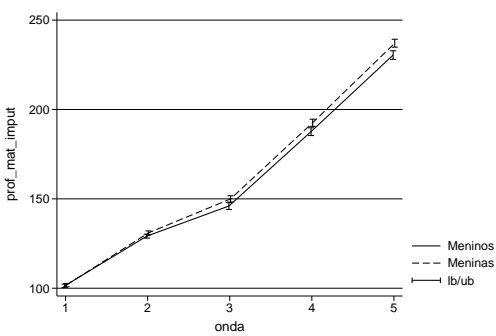

Matemática

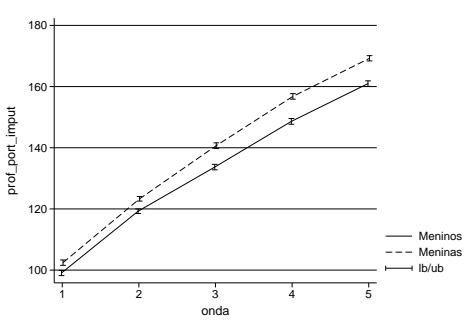

Português

Figura 5: Evolução da proficiência por gênero

a elevação é de mais de $153 \%$. Tal diferença pode ser explicada pela maior dificuldade em recuperar o atraso em matemática do que o atraso em português. Em função desse fato, uma parcela dos alunos, que entrou com uma habilidade em matemática mais baixa, pode ter uma dificuldade progressiva na aprendizagem, como bem esclarece Brooke et al. (2014).

Vale lembrar que a análise neste trabalho é apenas para os quatro primeiros anos do Ensino Fundamental. Se a tendência aqui encontrada continuar nos próximos anos do Ensino Fundamental e no Ensino Médio, a desigualdade em matemática irá atingir patamares ainda mais elevados ao final do Ensino Médio, o que é um alerta para a necessidade de investimentos nas crianças antes mesmo da entrada delas no Ensino Fundamental. Com isso, seria possível reduzir a desigualdade inicial e o atraso de parcela das crianças.

Tabela 3: Evolução da Desigualdade de Proficiência

\begin{tabular}{lcc}
\hline Onda & Matemática & Português \\
\hline 1 & 24,12 & 22,70 \\
2 & 29,14 & 19,76 \\
3 & 48,85 & 24,01 \\
4 & 55,41 & 24,59 \\
5 & 61,14 & 23,83 \\
Variação \% & 153,52 & 4,94 \\
\hline \multicolumn{2}{l}{ Fonte: GERES. }
\end{tabular}

Os próximos resultados se referem à desigualdade de oportunidades. Os cálculos foram realizados utilizando a técnica descrita na seção metodológica. O primeiro exercício realizado estima uma regressão para cada circunstância separadamente, fornecendo a contribuição bruta de cada circunstância. A tabela 4 mostra os resultados para as quatro circunstâncias para as proficiências em matemática e português.

Em relação à proficiência de matemática, a primeira onda apresenta o nível socioeconômico com a maior contribuição para a desigualdade de habilidades $(4,4 \%)$, seguida pelo município $(2,5 \%)$, raça $(0,8 \%)$ e gênero, sendo que esta última com praticamente nenhum efeito na desigualdade. A maior contribuição do nível socioeconômico está de acordo com a literatura da educação, que mostra que essa é a característica de maior impacto no aprendizado das crianças e também apresenta o maior impacto na desigualdade de notas (Diaz 2012, Ferreira \& Gignoux 2014, Gamboa \& Waltenberg 2012). 
Ao analisar a evolução da contribuição de cada circunstância, nota-se um aumento no efeito da raça, nível socioeconômico e gênero e uma diminuição apenas no efeito do município. O maior aumento foi percebido pelo gênero da criança, com mais de $5.000 \%$ de elevação na contribuição. No entanto, continua sendo a circunstância com menor contribuição na quinta onda, apenas $0,3 \%$. A contribuição da raça subiu praticamente $166 \%$, passando de menos de $1 \%$ para mais de $2 \%$ e ocupando a segunda posição no ranking de maior contribuição. Já a contribuição do nível socioeconômico subiu em torno de $55 \%$, passando a contribuir com quase $7 \%$ na desigualdade de matemática. $\mathrm{Na}$ última onda, o ranking de contribuição ficou com o nível socioeconômico em primeiro lugar $(6,8 \%)$, seguido da raça $(2,1 \%)$, município $(1,4 \%)$ e gênero $(0,3 \%)$.

Para a proficiência em português o ranking de contribuição na primeira onda aparece com o município em primeiro lugar $(19,4 \%)$, seguido pelo nível socioeconômico $(4,8 \%)$, o gênero $(0,6 \%)$ e pela raça $(0,05 \%)$. Apesar do município aparecer com a maior contribuição na primeira onda, essa contribuição diminui de forma significativa ao longo dos quatros anos, e, na quinta onda, tem a menor contribuição entre as circunstâncias analisadas $(1,6 \%)$, representando uma queda de quase $85 \%$. O nível socioeconômico tem um aumento de sua contribuição em mais de $40 \%$, raça um aumento de mais de $377 \%$ e gênero de quase $450 \%$. Ao final do período analisado, o ranking de contribuição para a desigualdade em português ficou com o nível socioeconômico em primeiro lugar $(6,8 \%)$, seguido do gênero $(3,1 \%)$, raça $(2,3 \%)$ e por último o município $(1,6 \%)$.

Tabela 4: Desigualdade de oportunidades - fatores individuais

\begin{tabular}{|c|c|c|c|c|c|c|c|c|}
\hline \multirow[b]{2}{*}{ Raça } & \multicolumn{4}{|c|}{ Matemática } & \multicolumn{4}{|c|}{ Português } \\
\hline & Nse & Mun & Sexo & Raça & Nse & Mun & Sexo & \\
\hline Onda 1 & 0,008 & 0,044 & 0,025 & 0,000 & 0,005 & 0,048 & 0,104 & 0,006 \\
\hline Onda 2 & 0,012 & 0,041 & 0,021 & 0,001 & 0,009 & 0,061 & 0,043 & 0,011 \\
\hline Onda 3 & 0,016 & 0,061 & 0,025 & 0,002 & 0,015 & 0,066 & 0,034 & 0,021 \\
\hline Onda 4 & 0,019 & 0,061 & 0,022 & 0,002 & 0,021 & 0,065 & 0,018 & 0,028 \\
\hline Onda 5 & 0,021 & 0,068 & 0,014 & 0,003 & 0,023 & 0,068 & 0,016 & 0,031 \\
\hline Variação \% & 165958 & 55175 & -45401 & 5396515 & 377638 & 40990 & -84676 & 449536 \\
\hline
\end{tabular}

A tabela 5 apresenta os resultados da desigualdade de oportunidades considerando todas as circunstâncias conjuntamente, tanto para matemática quanto para português. São apresentadas as medidas para a desigualdade de oportunidades (R2) e para a contribuição marginal de cada circunstância, calculada pela decomposição de shapley-shorroks, para cada onda e a variação percentual entre a quinta e primeira onda.

Na primeira onda, a desigualdade de oportunidades em matemática era de $7,4 \%$, enquanto que para português era de $16,8 \%$. Uma análise da contribuição de cada circunstância, aponta que essa diferença se dá principalmente pelo efeito do município, que em português é de 10,8\% e em matemática 2,5\%. A evolução da desigualdade de oportunidades ao longo dos anos é distinta para as duas disciplinas. Enquanto que, para matemática, nota-se um crescimento de mais de $35 \%$, em português, ocorre uma redução de mais de $20 \%$. Mesmo com movimentos em sentidos opostos, ao final da quinta onda, a de- 
sigualdade de oportunidades ainda é maior em português $(13,3 \%)$ do que em matemática $(10 \%)$. No entanto, na quinta onda, a diferença entre as duas disciplinas é explicada basicamente pelo gênero das crianças. Essa diferença entre os gêneros está de acordo com o que é apontado na literatura educacional e também com a tendência que já se delineava na figura 5. As meninas apresentam um aprendizado em português superior aos meninos, ao passo que, em matemática, essa diferença é menos significativa. ${ }^{6}$

É interessante comparar os resultados das contribuições de cada circunstância quando calculadas de forma bruta (tabela 4) de seus efeitos marginais (tabela 5). As contribuições do município de residência e do gênero não se alteram nas duas situações. Entretanto, as variáveis de raça e nível socioeconômico dimimuem sua contribuição para a desigualdade, tanto na proficiência de matemática quanto na proficiência de português. Essa redução indica que existe uma correlação entre estas variáveis. Esse fato está de acordo com a literatura de desigualdade, que mostra que dentro do grupo de negros, o percentual de pessoas com baixo nível socioeconômico é maior do que entre o grupo de brancos. Apesar da redução do efeito da raça quando controlado pelo nível socioeconômico, ainda resta um efeito de quase $2 \%$ nas duas disciplinas. Este resultado é importante, pois chama a atenção para um problema de discriminação racial no sistema educacional brasileiro. Ainda mais preocupante é o fato que o efeito da raça na desigualdade cresce de forma abrupta nos primeiros anos escolares, em torno de $148 \%$ para matemática e $303 \%$ para português. Com os resultados obtidos neste artigo, não é possível determinar exatamente de que forma essa discriminação ocorre, se dentro da escola, se no acesso à melhores escolas ou se em características fora da escola. Soares \& Alves (2003) e Soares et al. (2005) encontram resultados no mesmo sentido, mesmo controlando pelo nível socioeconômico, o desempenho dos negros é inferior ao desempenho dos brancos na escola.

É interessante examinar a desigualdade de oportunidades sem considerar o efeito do município, dado que a amostra deste estudo contém apenas quatro municípios e, portanto, pode enviesar os resultados. A tabela 6 apresenta os resultados para as estimações sem a circunstância município. As contribuições marginais de cada circunstância não sofrem alterações significativas em relação ao que foi apresentado na tabela 5. No entanto, a medida de desigualdade de oportunidades e, principalmente, sua evolução sofrem alterações interessantes. A desigualdade de oportunidades é inferior ao observado na tabela 5, em função de não contar mais com a contribuição do município. Já a evolução da desigualdade é crescente para as duas disciplinas, com um crescimento em torno de $80 \%$ para matemática e $108 \%$ para português.

\section{Considerações Finais}

O artigo avalia a evolução da desigualdade geral e de oportunidades no aprendizado de uma amostra de crianças durante os quatro primeiros anos do ensino fundamental. Ao utilizar uma base de dados longitudinal o artigo con-

\footnotetext{
${ }^{6} \mathrm{Na}$ literatura educacional, os meninos aparecem com um rendimento em matemática superior ao das meninas, embora em menor magnitude que a diferença apresentada em português. Entretanto, no banco de dados utilizado neste estudo, as meninas aparecem com um resultado ligeiramente superior ao dos meninos. Apesar desta inversão entre os gêneros no resultado de matemática, o menor efeito do gênero na desigualdade de matemática está de acordo com o encontrado em outros estudos Gamboa \& Waltenberg (2012)
} 
Tabela 5: Desigualdade de oportunidade - nse, raça, sexo e município

\begin{tabular}{|c|c|c|c|c|c|c|c|c|c|}
\hline Onda & $\mathrm{r} 2$ & nse & mun & sexo & raca & nse $\%$ & mun $\%$ & sexo $\%$ & raça $\%$ \\
\hline \multicolumn{10}{|c|}{ Matemática } \\
\hline 1 & 0,074 & 0,041 & 0,025 & 0,000 & 0,007 & 56295 & 34215 & 0,130 & 9360 \\
\hline 2 & 0,072 & 0,039 & 0,022 & 0,001 & 0,010 & 54363 & 30311 & 1513 & 13814 \\
\hline 3 & 0,102 & 0,060 & 0,027 & 0,002 & 0,014 & 58687 & 26077 & 1840 & 13396 \\
\hline 4 & 0,099 & 0,058 & 0,023 & 0,002 & 0,016 & 58741 & 22914 & 2298 & 16047 \\
\hline 5 & 0,100 & 0,065 & 0,014 & 0,003 & 0,017 & 65534 & 14027 & 3206 & 17233 \\
\hline Variação \% & 35180 & 57365 & -44580 & 3244057 & 148881 & 16411 & -59003 & 2373783 & 84111 \\
\hline \multicolumn{10}{|c|}{ Português } \\
\hline 1 & 0,168 & 0,049 & 0,108 & 0,006 & 0,005 & 29242 & 64447 & 3554 & 2757 \\
\hline 2 & 0,126 & 0,061 & 0,046 & 0,011 & 0,008 & 48592 & 36350 & 8874 & 6185 \\
\hline 3 & 0,135 & 0,066 & 0,035 & 0,022 & 0,012 & 48687 & 25818 & 16476 & 9019 \\
\hline 4 & 0,129 & 0,064 & 0,019 & 0,029 & 0,018 & 49436 & 14457 & 22168 & 13939 \\
\hline 5 & 0,133 & 0,067 & 0,016 & 0,031 & 0,019 & 50428 & 12065 & 23517 & 13990 \\
\hline Variação \% & -20496 & 37106 & -85116 & 426027 & 303418 & 72451 & -81279 & 561637 & 407420 \\
\hline
\end{tabular}

Tabela 6: Desigualdade de oportunidades - nse, raça e sexo

\begin{tabular}{lccccccc}
\hline Onda & r2 & Raça & nse & Sexo & Raça \% & nse \% & Sexo \% \\
\hline \multicolumn{7}{c}{ Matemática } \\
\hline 1 & 0,047 & 0,006 & 0,041 & 0,000 & 13549 & 86243 & 0,208 \\
2 & 0,049 & 0,010 & 0,038 & 0,001 & 19710 & 77996 & 2294 \\
3 & 0,074 & 0,014 & 0,058 & 0,002 & 18436 & 79065 & 2499 \\
4 & 0,076 & 0,016 & 0,058 & 0,002 & 20714 & 76312 & 2974 \\
5 & 0,085 & 0,017 & 0,064 & 0,003 & 20476 & 75716 & 3808 \\
Variação \% & 80350 & 172556 & 58336 & 3200035 & 51127 & -12206 & 1729797 \\
\hline \multicolumn{7}{l}{ Fonte: GERES. }
\end{tabular}

tribui para a literatura da economia da educação avaliando o comportamento das desigualdades educacionais nos primeiros anos da escola, bem como as desigualdades de oportunidades. Com isso, é possível investigar o papel da escola no processo de geração/redução das desigualdades de habilidades entre os indivíduos. Essa análise foi realizada utilizando o arcabouço teórico da Igualdade de Oportunidades. Para mensurar as habilidades, foram utilizados os níveis de proficiência em matemática e português. Os resultados encontrados diferem entre as proficiências em matemática e português.

Os resultados devem ser analisados considerando três aspectos: desigualdade geral; desigualdade de oportunidades; e efeito marginal de cada circunstância. Em relação à desigualdade geral, destaca-se o aumento das desigualdades em matemática, enquanto que para português houve relativa estabilidade.

Os resultados para a desigualdade de oportunidades variam em função das circuntâncias consideradas. Quando consideradas as quatro circunstâmcias conjuntamente, nível socioeconômico, gênero, raça e município de residência, a desigualdade de oportunidades em matemática cresce de aproximadamente $7 \%$ para $10 \%$, enquanto que para português os valores se alteram de quase $17 \%$ para um pouco mais de $13 \%$. Mas, apesar da desigualdade de oportunidades ser mais elevada para português, a taxa de crescimento desta desigualdade é positiva em matemática, cerca de $35 \%$, enquanto que para português observa-se uma redução de pouco mais de $20 \%$. No entanto, a queda na taxa 
de desigualdade de oportunidades em português é impulsionada pelo efeito do município. Em função disso e da pouca representatividade do município na amostra utilizada, também foi testada uma especificação retirando o município da análise, mantendo as outras três circunstâncias. Nessa especificação, o valor da desigualdade de oportunidades é menor para as duas disciplinas, um pouco mais de $8 \%$ para matemática e de $11 \%$ para português. No entanto, as duas disciplinas apresentam um aumento nos valores ao longo dos quatro anos, sendo que português apresenta um crescimento superior ao de matemática, $109 \%$ e $80 \%$, respectivamente.

Quando analisadas as circunstâncias isoladamente, o nível socioeconômico aparece como o fator de maior contribuição para a desigualdade educacional, reforçando evidências da literatura educacional que indicam que as características da família são os principais fatores determinantes do sucesso educacional. O município se mostra um fator importante no inicío do período escolar, no entanto, sua influência sobre a desigualdade educacional vai diminuindo ao longo do período escolar. Este resultado deve ser analisado com cautela, pois, nele estudo apenas quatro municípios fazem parte da amostra. A raça e o gênero da criança apresentam uma característica em comum, iniciam o período escolar influenciando pouco na desigualdade, tanto em matemática quanto em português, mas apresentam alta taxas de crescimento ao longo dos quatro anos. Vale lembrar que a análise é restrita aos quatro anos da amostra, ou seja, se a tendência de crescimento se mantiver no restante do período escolar, estes fatores irão explicar boa parte da desigualdade educacional.

Em uma perspectiva de políticas públicas, os resultados apresentados no artigo ressaltam a importância de investimentos na primeira infância para reduzir as desigualdades educacionais logo no início do período escolar. Pois, se a escola recebe crianças com uma diferença de habilidades muito acentuada, políticas educacionais voltadas apenas para melhorias de insumos escolares serão insuficientes para reduzir a desigualdade e principalmente a desigualdade de oportunidades.

\section{Referências Bibliográficas}

Albernaz, A., Ferreira, F. \& Franco, C. (2002), 'Qualidade e equidade no ensino fundamental brasileiro', Pesquisa e Planejamento Econômico 32(3).

Arneson, R. (1989), 'Equality and equal opportunity for welfare', Philosophical studies 56(1), 77-93.

Arneson, R. (1990), 'Liberalism, distributive subjectivism, and equal opportunity for welfare', Philosophy \& public affairs pp. 158-194.

Baker, F. \& Kim, S. (2004), Item response theory: Parameter estimation techniques, CRC Press.

Barro, R. (2000), 'Inequality and growth in a panel of countries', Journal of economic growth $\mathbf{5}(1), 5-32$.

Barros, R. d., Foguel, M. \& Ulyssea, G. (2006), Desigualdade de renda no brasil: uma análise da queda recente, in 'Desigualdade de renda no Brasil: uma análise da queda recente', Ipea. 
Barros, R. d. \& Mendonça, R. (1995), 'Os determinantes da desigualdade no brasil'.

Betts, J. \& Roemer, J. (2005), 'Equalizing opportunity for racial and socioeconomic groups in the united states through educational finance reform'.

Bonelli, R. \& Sedlacek, G. (1988), 'Distribuição de renda: evolução no último quarto de século'.

Bourguignon, F. (1981), 'Pareto superiority of unegalitarian equilibria in stiglitz'model of wealth distribution with convex saving function', Econometrica: Journal of the Econometric Society pp. 1469-1475.

Brooke, N., da Silva Fernandes, N., de Miranda, I. \& Soares, T. (2014), 'Modelagem do crescimento da aprendizagem nos anos iniciais com dados longitudinais da pesquisa geres', Educ. Pesqui 40(1), 77-94.

Checchi, D. \& Peragine, V. (2005), Regional disparities and inequality of opportunity: the case of italy, Technical report, IZA Discussion Papers.

Checchi, D. \& Peragine, V. (2010), 'Inequality of opportunity in italy', The Journal of Economic Inequality 8(4), 429-450.

Cohen, G. (1989), 'On the currency of egalitarian justice', Ethics pp. 906-944.

Deininger, K. \& Squire, L. (1996), 'A new data set measuring income inequality', The World Bank Economic Review 10(3), 565-591.

Deininger, K. \& Squire, L. (1998), 'New ways of looking at old issues: inequality and growth', Journal of development economics 57(2), 259-287.

Diaz, M. (2012), '(des)igualdades de oportunidades no ensino médio brasileiro: Escolas públicas e privadas', Economia 13(3a), 553-568.

Dworkin, R. (1981a), 'What is equality? part 1: Equality of welfare', Philosophy \& Public Affairs pp. 185-246.

Dworkin, R. (1981b), 'What is equality? part 2: Equality of resources', Philosophy \& Public Affairs pp. 283-345.

Ferreira, F. (2000), Os determinantes da desigualdade de renda no Brasil: luta de classes ou heterogeneidade educacional?, Pontifícia Universidade Católica de Rio de Janeiro, Departamento de Economia.

Ferreira, F. \& Gignoux, J. (2014), 'The measurement of educational inequality: Achievement and opportunity', The World Bank Economic Review 28(2), 210-246.

Ferreira, F. \& Litchfield, J. (1996), Growing apart: inequality and poverty trends in brazil in the 1980s, Technical report, Suntory and Toyota International Centres for Economics and Related Disciplines, LSE.

Ferreira, F. \& Litchfield, J. (1999), 'Educacion o inflacion?: Explicando la desigualdad en brasil en la decada de los ochenta', CÁRDENAS, M., LUSTIG, N.(eds.) . 
Ferreira, F., Walton, M. et al. (2005), World development report 2006: equity and development, Vol. 28, World Bank Publications.

Fishlow, A. (1972), 'Brazilian size distribution of income', The American Economic Review pp. 391-402.

Galor, O. \& Moav, O. (2004), 'From physical to human capital accumulation: Inequality and the process of development', The Review of Economic Studies 71(4), 1001-1026.

Gamboa, L. \& Waltenberg, F. (2012), 'Inequality of opportunity for educational achievement in latin america: Evidence from pisa 2006-2009', Economics of Education Review 31(5), 694-708.

Hoffman, R. (1989), 'Evolução da distribuição da renda no brasil, entre pessoas e entre famílias, 1979/86', Mercado de trabalho e distribuição de renda: uma coletânea. Série Monográfica 35.

Lam, D. \& Levison, D. (1990), 'Idade, experiência, escolaridade e diferenciais de renda: Estados unidos e brasil', Pesquisa e planejamento econômico 20(2), 219-256.

Langoni, C. (2005), Distribuição da renda e desenvolvimento econômico do Brasil, FGV Editora.

Marrero, G. \& Rodríguez, J. (2013), 'Inequality of opportunity and growth', Journal of Development Economics 104, 107-122.

Oliveira, L. (2008), Três investigações sobre escalas de proficiência e suas interpretações, PhD thesis, Tese (Doutorado em Educação)-Departamento de Educação, Pontifícia Universidade Católica do Rio de Janeiro, Rio de Janeiro.

Peragine, V. \& Serlenga, L. (2007), Higher education and equality of opportunity in italy, IZA Discussion Papers 3163, Institute for the Study of Labor (IZA).

URL: $h t t p: / / i d e a s . r e p e c . o r g / p / i z a / i z a d p s / d p 3163 . h t m l$

Pignataro, G. (2012), 'Equality of opportunity: Policy and measurement paradigms', Journal of Economic Surveys 26(5), 800-834.

Pinheiro, L., Oliveira Fontoura, N. \& Querino, A. C. (2009), Retrato das desigualdades de gênero e raça, 3ra ed. Brasilia: AECID; UNIFEM; IPEA.

Ramos, L. (1993), A distribuição de rendimentos no brasil: 1976/85, in 'IPEA', number 141, Ipea.

Ramos, X. (2012), 'Empirical approaches to inequality of opportunity: Principles, measures, and evidence'.

Rawls, J. (1971), 'A theory of justice'.

Rebelo, S. (1991), 'Long-run policy analysis and long-run growth', Journal of Political Economy 99(3), 500-521.

Riani, J. \& Rios-Neto, E. (2008), 'Background familiar versus perfil escolar do município: qual possui maior impacto no resultado educacional dos alunos brasileiros', Revista Brasileira de Estudos de População 25(2), 251-269. 
Roemer, J. (1998), Equality of opportunity, Cambridge Univ Press.

Roemer, J. E., Aaberge, R., Colombino, U., Fritzell, J., Jenkins, S. P., Lefranc, A., Marx, I., Page, M., Pommer, E., Ruiz-Castillo, J. et al. (2003), 'To what extent do fiscal regimes equalize opportunities for income acquisition among citizens?', Journal of Public Economics 87(3), 539-565.

Sen, A. (1979), 'Equality of what?'.

Soares, J. \& Alves, M. T. (2003), 'Desigualdades raciais no sistema brasileiro de educação básica', Educação e Pesquisa 29(1), 147-165.

Soares, J. \& Oliveira, F. (2009), O cálculo de nível socioeconômico (nse) dos alunos do projeto geres, Technical report, Universidade Federal de Minas Gerais.

Soares, S., Beltrão, K., Barbosa, M. \& Ferrão, M. E. (2005), Os mecanismos de discriminação racial nas escolas brasileiras, in 'Os Mecanismos de discriminação racial nas escolas brasileiras', Ipea.

Waltenberg, F. \& Vandenberghe, V. (2007), 'What does it take to achieve equality of opportunity in education?: An empirical investigation based on brazilian data', Economics of Education Review 26(6), 709-723. 